\title{
Network-based identification of genetic factors in Ageing, lifestyle and Type 2 Diabetes that Influence in the progression of Alzheimer's disease
}

\author{
Utpala Nanda Chowdhury ${ }^{1}$, Shamim Ahmad ${ }^{1}$, M. Babul Islam ${ }^{2}$, Fazlul Huq ${ }^{3}$, \\ Julian M.W. Quinn ${ }^{4}$ and Mohammad Ali Moni ${ }^{3,4, *}$ \\ ${ }^{1}$ Dept. of Computer Science and Engineering, University of Rajshahi, Rajshahi, Bangladesh, \\ ${ }^{2}$ Dept. of Applied Physics and Electronic Engineering, University of Rajshahi, Rajshahi, Bangladesh, \\ ${ }^{3}$ Decipline of Biomedical Science, Faculty of Medicine and Health, The University of Sydney, NSW, Australia and \\ ${ }^{4}$ Garvan Institute of Medical Research, University of New South Wales, Sydney, Australia. \\ *mohammad.moni@sydney.edu.au \\ Associate Editor: XXXXXXX \\ Received on XXXXX; revised on XXXXX; accepted on XXXXX
}

\begin{abstract}
Motivation: Alzheimer's disease (AD) is currently incurable and the causative risk factors are still poorly understood, which impedes development of effective prevention and treatment strategies. We propose a network-based quantitative framework to reveal details of the complex interaction between the various genetic contributors to $A D$ susceptibility. We analyzed gene expression microarray data from tissues affected by AD, advanced ageing, high alcohol consumption, type II diabetes, high body fat, high dietary fat, obesity, high dietary red meat intake, sedentary lifestyle, smoking, and control datasets. We developed genetic associations and diseasome networks for these factors and $A D$ using the neighborhood-based benchmarking and multilayer network topology approaches.

Results: The study identified 484 genes differentially expressed between AD and controls. Among these, 27 genes showed elevated expression both in individuals in $A D$ and in smoker datasets; similarly 21 were observed in $A D$ and type II diabetes datasets and 12 for $A D$ and sedentary lifestyle datsets. However, $A D$ shared less than ten such elevated expression genes with other factors examined. 3 genes, namely HLA-DRB4, IGH and IGHA2 showed increased expression among the AD, type II diabetes and alcohol consumption datasets; 2 genes, IGHD and IGHG1, were commonly up-regulated among the AD, type II diabetes, alcohol consumption and sedentary lifestyle datasets. Protein-protein interaction networks identified 10 hub genes: CREBBP, PRKCB, ITGB1, GAD1, GNB5, PPP3CA, CABP1, SMARCA4, SNAP25 and GRIA1. Ontological and pathway analyses genes, including Online Mendelian Inheritance in Man (OMIM) and dbGaP databases were used for gold benchmark gene-disease associations to validate the significance of these putative target genes of AD progression.

Conclusion: Our network-based methodologies have uncovered molecular pathways that may influence $A D$ development, suggesting novel mechanisms that contribute to $A D$ risk and which may form the basis of new therapeutic and diagnostic approaches.

Contact: mohammad.moni@sydney.edu.au
\end{abstract}

\section{Introduction}

Alzheimer's disease (AD) is the most common form of dementia and is characterized by gradual degeneration in memory, cognitive processes, language use, and learning capacity Duthey (2013); Rahman et al. 
“output" — 2018/12/5 - 2:26 — page $2-\# 2$

(2018b). Initial indications begin with a reduced ability to retain recent memories, but with progression all cognitive functions are inevitably affected, resulting in complete dependency for basic daily activities and greatly increased risk of premature death Serrano-Pozo et al. (2011); Rahman et al. (2018c). AD is irremediable that accounts for $60 \%$ to $80 \%$ of all dementia cases and estimated to affect over 24 million people worldwide. In the United States, 93,541 deaths resulting from AD were officially recorded in 2014, which is ranked sixth among all causes of death in the United States and fifth among all causes of death after 65 years of age. The premature death rate of $\mathrm{AD}$ sufferers increased by $89 \%$ within the five years up to 2010, whereas death rates associated with other major morbidities such as cardiac disease, stroke, breast and prostate cancer, and AIDS all declined in that frame. Currently, one new case of the AD is developed in every 66 seconds, a rapid rate of development expected to double by 2050 Association et al. (2017); Rahman et al. (2018a)

The pathogenesis of the AD is not clearly understood, but it is hypothesized that both genetic and environmental factors are the primary causes. Genes encoding amyloid precursor protein (APP), presenilin 1 (PSEN1) and presenilin 2 (PSEN2) have been identified as associated with AD development Waring and Rosenberg (2008). Age is found to be the most influential risk factor for $\mathrm{AD}$, along with a sedentary lifestyle. Typically $\mathrm{AD}$ develops after the age of 65 years and almost half individuals ove 85 years old have AD Lindsay et al. (2002). Obesity also increases the risk of dementia and eventually AD Kivipelto et al. (2005). Type II diabetes, hypertension, smoking and dietary fats can increase the risk of developing AD Mayeux and Stern (2012) Janson et al. (2004) Morris et al. (2003); Rahman et al. (2018a). Meta-analysis of prospective studies suggests that alcohol consumption in late life yields reduced the risk of dementia and hence reduced the risk of $\mathrm{AD}$ Anstey et al. (2009). AD is a complex polygenic disorder, and many of the associated factors are yet to be identified. For these reasons, there are many problems with accurate diagnosis, characterizing heterogeneous groups of patients who may respond differently to treatment and complicate decisions regarding effective treatment. With such poor understanding of a disease the discovery of further genetic factors could be an importan avenue to development of improved diagnostic profiles, and a cleare understanding of the disease process Tilley et al. (1998). The key genetic factors associated with susceptibility to complex diseases can be effectively unravelled by genome-wide association studies, and the usefulness of this approach has been proven empirically. Our methodology employed here aims to identify genetic factors influencing common and complex conditions against the background of the random variation seen in population as a whole Altshuler et al. (2008); Moni and Liò (2015).

Molecular association analyses, including differential gene expression determination, protein-protein interactions (PPIs), gene ontologies (GO) and metabolic pathways can ascribe gene activity-based relationship between AD and various risk factors for the disease Rzhetsky et al. (2007) Moni and Liò (2014a). Differentially expressed transcripts seen in studies comparing control individuals with individual affected by a disease (or disease risk factor) identify putative disease-associated genes of interest; a differentially expressed gene identified in $\mathrm{AD}$ can be more strongly linked to $\mathrm{AD}$ when it is shared with $\mathrm{AD}$ risk factor differentially expressed genes Goh et al. (2007) Feldman et al. (2008); Moni et al. (2018). From proteomics point of view, genes are also associated through biological modules such as PPIs, gene ontologies or molecular pathways Lage et al. (2007) Suthram et al. (2010); Satu et al. (2018).

Recently there has been many advances in network-based integrative analytical methods used by researchers to identify possible roles of biomolecules in complex diseases Moni and Liò (2014b) Moni and Lio (2017) Torkamani et al. (2008). A number of transcriptomic and genetic studies have been conducted on AD Bertram et al. (2010) Logue et al. (2011) Seshadri et al. (2010); Hossain et al. (2018a). However, most of these findings have been limited at the transcript level, since the functional interactions among the gene products have not commonly been considered. Since biological molecules interact with each other to carry out functions in biological processes in cells and tissues, integrative analysis within network medicine context is essential to understand the molecular mechanisms behind diseases and to identify critical biomolecules. Thus, we have used a network-based analysis to determine the genetic influence of associated risk factors and disorders for AD progression, including studies of gene expression profiling, PPI sub-network, gene ontologies and molecular pathways. An extensive study regarding phylogenetic and pathway analysis was therefore conducted to reveal the genetic associations of the AD. Significance of these genes and pathways in AD processes were also validated with gold benchmarking datasets including Online Mendelian Inheritance in Man (OMIM) and dbGaP gene-disease associations databases.

\section{Materials and Methods}

\subsection{Data}

We have analyzed gene expression microarray datasets to identify the association of different factors with the $\mathrm{AD}$ at the molecular level. All the datasets used in this study were collected from the National Center for Biotechnology Information (NCBI) Gene Expression Omnibus database (https://www.ncbi.nlm.nih.gov/geo/). Ten different datasets with accession numbers: GSE1297, GSE23343, GSE15524, GSE25941, GSE1786, GSE68231, GSE6573, GSE25220, GSE52553, and GSE4806 were analyzed for this study Blalock et al. (2004) MacLaren et al. (2010) Raue et al. (2012) Radom-Aizik et al. (2005) Kakehi et al. (2015) Misu et al. (2010) Herse et al. (2007) Hebels et al. (2011) McClintick et al. (2014) Büttner et al. (2007); Hossain et al. (2018b). The AD dataset (GSE1297) is obtained by gene expression profiling of hippocampal tissues on 31 separate microarrays from nine control subjects and $22 \mathrm{AD}$ patients with varying severity. The type II diabetes dataset (GSE23343) contains gene expression data obtained through extensive analysis after conducting liver biopsies in humans. The source of the obesity dataset (GSE15524) is subcutaneous and omental adipose tissue analyzed through expression profiling of 20,000 probes in 28 tissue samples. The advanced age dataset (GSE25941) consists of a global microarray data from skeletal muscle transcriptome of 28 different subjects. The sedentary lifestyle dataset (GSE1786) was obtained by expression profiling array from the vastus lateralis muscle using needle biopsies. The high-fat diet (HFD) dataset (GSE68231) is the expression data from human skeletal muscle identifying accumulation of intramyocellular lipid (IMCL). The high body fat (HBF) dataset (GSE6573) is an Affymetrix human gene expression array data from the abdominal fat tissue. The red meat dietary intervention dataset (GSE25220) is an Agilent-014850 whole human genome microarray data from human colon biopsies before and after participating in a high redmeat dietary intervention. The alcohol consumption dataset (GSE52553) is an Affymetrix human gene expression array data of Lymphoblastoid cells from 21 alcoholics and 21 control subjects. The smoking datase (GSE4806) is a gene expression profiles of T-lymphocytes from smokers and non-smokers.

\subsection{Method}

Analyzing oligonucleotide microarray data for gene expression is known to be an effective and responsive approach to identify new molecular determinants of human diseases. In this study, we used this methodology along with global transcriptome analysis to investigate the gene expression profiles of the $\mathrm{AD}$ with 8 risk factors and type II diabetes. To mitigate the problems involving messenger RNA (mRNA) data comparison using 
“output" — 2018/12/5 - 2:26 — page 3 - \#3

different platforms and experimental set-ups, we normalized each gene expression data for each disease using the Z-score (or zero mean) transformation for both disease and control state Sakib et al. (2018). Each sample of gene expression matrix was normalized using mean and standard deviation. The expression value of gene $i$ in sample $j$ represented by $g_{i j}$ was transformed into $Z_{i j}$ by computing

$$
Z_{i j}=\frac{g_{i j}-\operatorname{mean}\left(g_{i}\right)}{S D\left(g_{i}\right)}
$$

where $\mathrm{SD}$ is the standard deviation. Comparing values of gene expression for various samples and diseases are made possible by this transformation. Data were transformed using $\log _{2}$ and differentially expressed genes for both disease and control states were obtained by performing unpaired student t-test, and significant genes were identified by using threshold values. A threshold for $\mathrm{p}$-value and absolute base two log fold change $(\log \mathrm{FC})$ values were set to at most 0.05 and at least 1.0 respectively. We built two infectome-diseasome relationships networks using Cytoscape (v3.5.1) Smoot et al. (2010); Moni et al. (2014) for both up-regulated and down-regulated genes focusing on the AD. Each node of the networks are either diseases or associative factors. These networks can also be considered as bipartite graphs where diseases or factors are connected when they share at least 1 differentially expressed gene.

We used the web-based visualization software STRING Szklarczyk et al. (2016) for the construction and analysis of the Protein-Protein Interaction (PPI) network which was further analyzed by Cytoscape. An undirected graph representation was used for the PPI network, where the nodes indicate proteins and the edges symbolize the interactions between the proteins. We performed a topological analysis using Cyto-Hubba plugin Chen et al. (2009) to identify highly connected proteins (i.e., hub proteins) in the network and the degree metrics were employed Calimlioglu et al. (2015); Xu et al. (2015). For further introspection into the metabolic pathways of the $\mathrm{AD}$, we incorporated the pathway and gene ontology analysis on all the differentially expressed genes that were common between the $\mathrm{AD}$ and the other risk factors datasets using the web-based gene set enrichment analysis tool EnrichR Kuleshov et al. (2016). In this analysis, the Gene Ontology (GO) Biological Process (BP) and KEGG pathway databases were selected as annotation sources. For statistical significance, the highest adjusted p-value was considered 0.05 to obtain enrichment results. Obtained GO and pathway were further analyzed by Cytoscape. Moreover, two gold bench mark validated datasets, OMIM (https://www.omim.org/) and dbGaP (https://www.ncbi.nlm.nih.gov/gap) were included in our study to validate the principle of our network based approach.

\section{Results}

\subsection{Gene Expression Analysis}

To identify dysregulated genes linked to $\mathrm{AD}$ the gene expression patterns from hippocampal CA1 tissues of $\mathrm{AD}$ patients were analyzed and compared with normal subject using the NCBI GEO2R online tool (https://www.ncbi.nlm.nih.gov/geo/geo2r/?acc=GSE1297) Blalock et al. (2004). 484 genes (p-value at most 0.05 and absolute $\log _{2}$ fold change value at least 1.0) were found to be differentially expressed compared to healthy subjects where 336 genes were up-regulated and 148 genes were down-regulated.

In order to investigate the relationship of the $\mathrm{AD}$ with 8 risk factors and type II diabetes, we performed several steps of statistical analysis for mRNA microarray data regarding each risk factors and disease. Thus, we selected the most significant over and under regulated genes for each risk factor and disease. Our analysis identified a large number of dysregulated genes, namely 958 genes in advanced ageing, 1405 in high alcohol consumption,
824 in high body fat (HBF), 739 in high fat diet (HFD), 381 in obesity, 482 in high dietary red meat, 800 in sedentary lifestyle, 400 in smoking and 1438 in diabetes type II datasets.

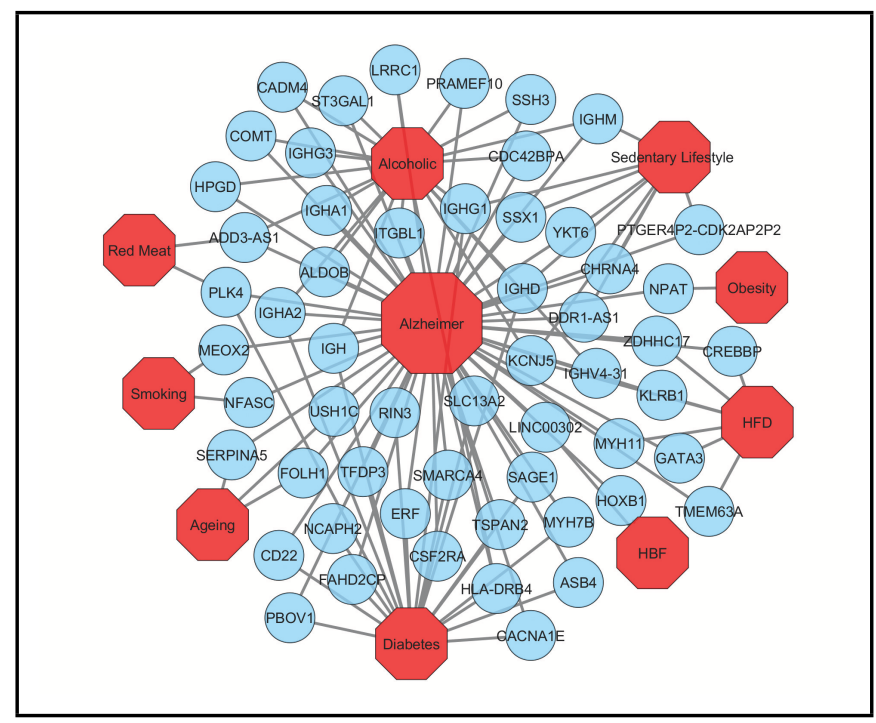

Fig. 1. Diseasome network of the $\mathrm{AD}$ with type II diabetes, ageing, sedentary lifestyle, HFD, HBF, high dietary red meat, high alcohol consumption, obesity and smoking. Redcolored octagon-shaped nodes represent categories of factors and or disease, and roundshaped sky blue-colored nodes represent up-regulated genes that are common for the $\mathrm{AD}$ with the other risk factors and or diseases. A link is placed between a risk factor or disease and gene if alteration expression of that gene is associated with the specific disorder.

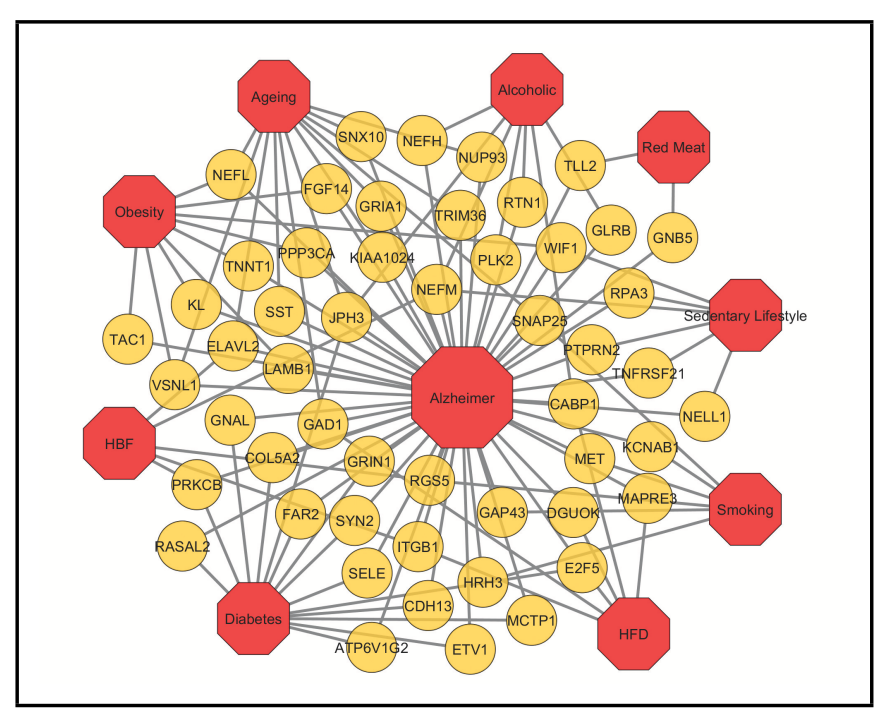

Fig. 2. Diseasome network of the AD with type II diabetes, ageing, sedentary lifestyle, HFD, HBF, dietary red meat, high alcohol consumption, obesity and smoking. Red-colored octagon-shaped nodes represent categories of factors and or disease, and round-shaped yellow-colored nodes represent down-regulated genes that are common for the $\mathrm{AD}$ with the other risk factors and or diseases. A link is placed between a risk factor or disease and gene if altered expression of that gene is associated with the specific disorder.

The over- and under- expressed genes identified as in common between $\mathrm{AD}$ and other risk factors and diseases were also detected through a cross-comparative analysis. The findings demonstrated that AD shares 
Table 1. Some significant KEGG pathways that are related to the nervous

a total of $35,34,18,15,13,10,8,7$ and 4 significant differentially expressed genes with type II diabetes, alcohol consumption, sedentary lifestyle, ageing, HFD, obesity, smoking, HBF and dietary red meat datasets respectively. Two infectome-diseasome associations network centered on the $\mathrm{AD}$ were built using Cytoscape to identify statistically significant associations among these risk factors and diseases. Network shown in Figure-1 interprets the association among up- regulated genes and another network shown in Figure-2 depicts relations between among down regulated genes. Notably, 3 significant genes, HLA-DRB4, IGH and IGHA2 are commonly up-regulated in the $\mathrm{AD}$, type II diabetes and alcohol consumption datasests; 2 significant genes IGHD and IGHG1 were commonly up regulated among the $\mathrm{AD}$, type II diabetes, alcohol consumption and sedentary lifestyle datasets. It is noteworthy that a relatively higher number of differentially expressed genes was identified as in common between the $\mathrm{AD}$ and type II diabetes datasets, whereas the $\mathrm{AD}$ and high dietary red meat shared only 4 differentially expressed genes.

\subsection{Protein-Protein Interaction Network Analysis}

The PPI network was constructed using all the distinct 108 (from total 144) differentially expressed genes that were identified as in common between the $\mathrm{AD}$ and other risk factors and disease datasets (Figure-3). Each node in the network represents a protein and an edge indicates the interaction between two proteins. The network is also grouped into 9 clusters representing risk factors and diseases to depict the protein links. It is notable that $\mathrm{KCNJ} 5$ protein belongs to the highest number (3) of clusters indicating that it is gene most commonly found among the $\mathrm{AD}$, alcohol consumption, HFD and sedentary lifestyle datasets and interacts with othe proteins from different clusters. In addition the protein products of PLK4 PRKCB, E2F5, GAD1, VSNL1, RGS5, ITGB1, CABP1 and NEFM belong to two clusters each, and interact with other proteins in the network For topological analysis, a simplified PPI network was constructed using Cyto-Hubba plugin to show 10 most significant hub proteins (Figure-4) which are CREBBP, PRKCB, ITGB1, GAD1, GNB5, PPP3CA, CABP1, SMARCA4, SNAP25 and GRIA1. The most significantly identified hub protein CREBBP (CREB binding protein) plays major role during the evolution of central nervous system. Alteration of CREBBP activity is known to be implicated in AD progression Rouaux et al. (2004).

\subsection{Pathway and Functional Correlation Analysis}

In order to identify the molecular pathways associated with the AD and predicted links to the affected pathways, we performed pathway analysis on all the differentially expressed genes that were common among the $\mathrm{AD}$ and other risk factors and diseases using the KEGG pathway database (http://www.genome.jp/kegg/pathway.html) and the web-based gene set enrichment analysis tool EnrichR Kuleshov et al. (2016). A total of 115 pathways were found to be over-represented among several groups. Notably, nine significant pathways that are related to the nervous system were found which are Long-term potentiation (hsa04720), Synaptic vesicle cycle (hsa04721), Retrograde endocannabinoid signaling (hsa04723), Glutamatergic synapse (hsa04724), Cholinergic synapse (hsa04725), Serotonergic synapse (hsa04726), GABAergic synapse (hsa04727) Dopaminergic synapse (hsa04728), and Long-term depression (hsa04730). These pathways along with some other common pathways found are shown in Table 1. A gene and pathway association is analyzed by constructing a network for the resulted pathways using Cytoscape (Figure-5).

We identified over-represented ontological groups by performing gene biological process ontology enrichment analysis using EnrichR on the commonly dysregulated genes between the $\mathrm{AD}$ and other risk factors and diseases. Total 215 significant gene ontology groups including peripheral nervous system neuron development (GO:0048935), neurotransmitter transport (GO:0006836), neuromuscular synaptic system and common among the $\mathrm{AD}$ and other risk factors and diseases. (Ag=Ageing, T2D=Type II Diabetes, Ob=Obesity, Sm=Smoking, RM=Red Meat, $\mathrm{AC}=$ Alcohol Consumption, $\mathrm{SL}=$ Sedentary Lifestyle.)

\begin{tabular}{|c|c|c|c|}
\hline KEGG ID & Pathway & Genes in pathway & $\begin{array}{l}\text { Risk } \\
\text { fac./dis. }\end{array}$ \\
\hline hsa04720 & $\begin{array}{l}\text { Long-term } \\
\text { potentiation }\end{array}$ & $\begin{array}{l}\text { GRIA1, PRKCB, } \\
\text { GRIN1, CREBBP, } \\
\text { PPP3CA }\end{array}$ & $\begin{array}{l}\mathrm{Ag}, \quad \mathrm{Ob}, \\
\mathrm{T} 2 \mathrm{D}, \mathrm{HBF}, \\
\text { HFD }\end{array}$ \\
\hline hsa05014 & $\begin{array}{l}\text { Amyotrophic } \\
\text { lateral sclerosis } \\
(\text { ALS) }\end{array}$ & $\begin{array}{ll}\text { GRIA1, } & \text { NEFL, } \\
\text { NEFM, } & \text { NEFH, } \\
\text { PPP3CA } & \end{array}$ & $\begin{array}{ll}\mathrm{Ag}, & \mathrm{AC}, \\
\mathrm{HBF}, & \mathrm{Ob}, \\
\mathrm{SL} & \end{array}$ \\
\hline hsa04728 & $\begin{array}{l}\text { Dopaminergic } \\
\text { synapse }\end{array}$ & $\begin{array}{l}\text { KCNJ5, } \\
\text { GNAL, } \\
\text { GNB5 }\end{array}$ & $\begin{array}{l}\text { AC, T2D, } \\
\text { HBF, RM }\end{array}$ \\
\hline hsa05031 & $\begin{array}{l}\text { Amphetamine } \\
\text { addiction }\end{array}$ & $\begin{array}{l}\text { GRIA1, PRKCB, } \\
\text { GRIN1, PPP3CA }\end{array}$ & $\begin{array}{l}\mathrm{Ag}, \mathrm{T} 2 \mathrm{D}, \\
\mathrm{HBF}, \mathrm{Ob}\end{array}$ \\
\hline hsa04662 & $\begin{array}{l}\text { B cell receptor } \\
\text { signaling pathway }\end{array}$ & $\begin{array}{l}\text { PRKCB, } \\
\text { PPP3CA }\end{array}$ & $\begin{array}{l}\text { T2D, HBF, } \\
\mathrm{Ob}\end{array}$ \\
\hline hsa04940 & Type I T2D mellitus & GAD1, PTPRN2 & $\begin{array}{l}\mathrm{Ag}, \mathrm{HFD}, \\
\mathrm{SL}\end{array}$ \\
\hline hsa05100 & $\begin{array}{l}\text { Bacterial invasion } \\
\text { of epithelial cells }\end{array}$ & ITGB1, MET & $\begin{array}{l}\text { HBF, } \\
\text { HFD, Sm }\end{array}$ \\
\hline hsa05140 & Leishmaniasis & $\begin{array}{l}\text { HLA-DRB4, } \\
\text { PRKCB, ITGB1 }\end{array}$ & $\begin{array}{l}\text { T2D, HBF, } \\
\text { HFD }\end{array}$ \\
\hline hsa05146 & Amoebiasis & $\begin{array}{l}\text { GNAL, PRKCB, } \\
\text { LAMB1, }\end{array}$ & $\begin{array}{l}\text { T2D, HBF, } \\
\mathrm{Ob}\end{array}$ \\
\hline hsa00250 & $\begin{array}{l}\text { Alanine, aspartate } \\
\text { and glutamate } \\
\text { metabolism }\end{array}$ & FOLH1, GAD1 & Ag, HFD \\
\hline hsa04014 & $\begin{array}{l}\text { Ras signaling } \\
\text { pathway }\end{array}$ & $\begin{array}{l}\text { PRKCB, RASAL2, } \\
\text { GRIN1, GNB5 }\end{array}$ & $\mathrm{T} 2 \mathrm{D}, \mathrm{RM}$ \\
\hline hsa04310 & $\begin{array}{l}\text { Wnt signaling } \\
\text { pathway }\end{array}$ & $\begin{array}{l}\text { PRKCB, PPP3CA, } \\
\text { WIF1 }\end{array}$ & $\mathrm{HBF}, \mathrm{Ob}$ \\
\hline hsa04360 & Axon guidance & ITGB1, MET & $\mathrm{HBF}, \mathrm{Sm}$ \\
\hline hsa04370 & $\begin{array}{l}\text { VEGF signaling } \\
\text { pathway }\end{array}$ & PRKCB, PPP3CA & $\mathrm{HBF}, \mathrm{Ob}$ \\
\hline hsa04512 & $\begin{array}{l}\text { ECM-receptor } \\
\text { interaction }\end{array}$ & ITGB1, LAMB1 & $\mathrm{HBF}, \mathrm{Ob}$ \\
\hline hsa04514 & $\begin{array}{l}\text { Cell adhesion } \\
\text { molecules (CAMs) }\end{array}$ & $\begin{array}{l}\text { HLA-DRB4, } \\
\text { SELE, CD22, } \\
\text { ITGB1 }\end{array}$ & $\mathrm{T} 2 \mathrm{D}, \mathrm{HBF}$ \\
\hline hsa04721 & $\begin{array}{l}\text { Synaptic vesicle } \\
\text { cycle }\end{array}$ & SNAP25, SNAP25 & $\mathrm{Ag}, \mathrm{Sm}$ \\
\hline hsa04723 & $\begin{array}{l}\text { Retrograde } \\
\text { endocannabinoid } \\
\text { signaling }\end{array}$ & PRKCB, GNB5 & $\mathrm{HBF}, \mathrm{RM}$ \\
\hline hsa04727 & $\begin{array}{l}\text { GABAergic } \\
\text { synapse }\end{array}$ & PRKCB, GNB5 & $\mathrm{HBF}, \mathrm{RM}$ \\
\hline hsa04730 & $\begin{array}{l}\text { Long-term } \\
\text { depression }\end{array}$ & GRIA1, PRKCB & $\mathrm{Ag}, \mathrm{HBF}$ \\
\hline
\end{tabular}

transmission (GO:0007274), peripheral nervous system development (GO:0007422), negative regulation of neurological system process (GO:0031645), regulation of neurotransmitter secretion (GO:0046928), regulation of neuronal synaptic plasticity (GO:0048168), autonomic nervous system development (GO:0048483), sympathetic nervous system development (GO:0048485), neuromuscular process controlling balance (GO:0050885), neuron apoptotic process (GO:0051402), regulation 
$\bigoplus$

“output" — 2018/12/5 — 2:26 — page 5 — \#5

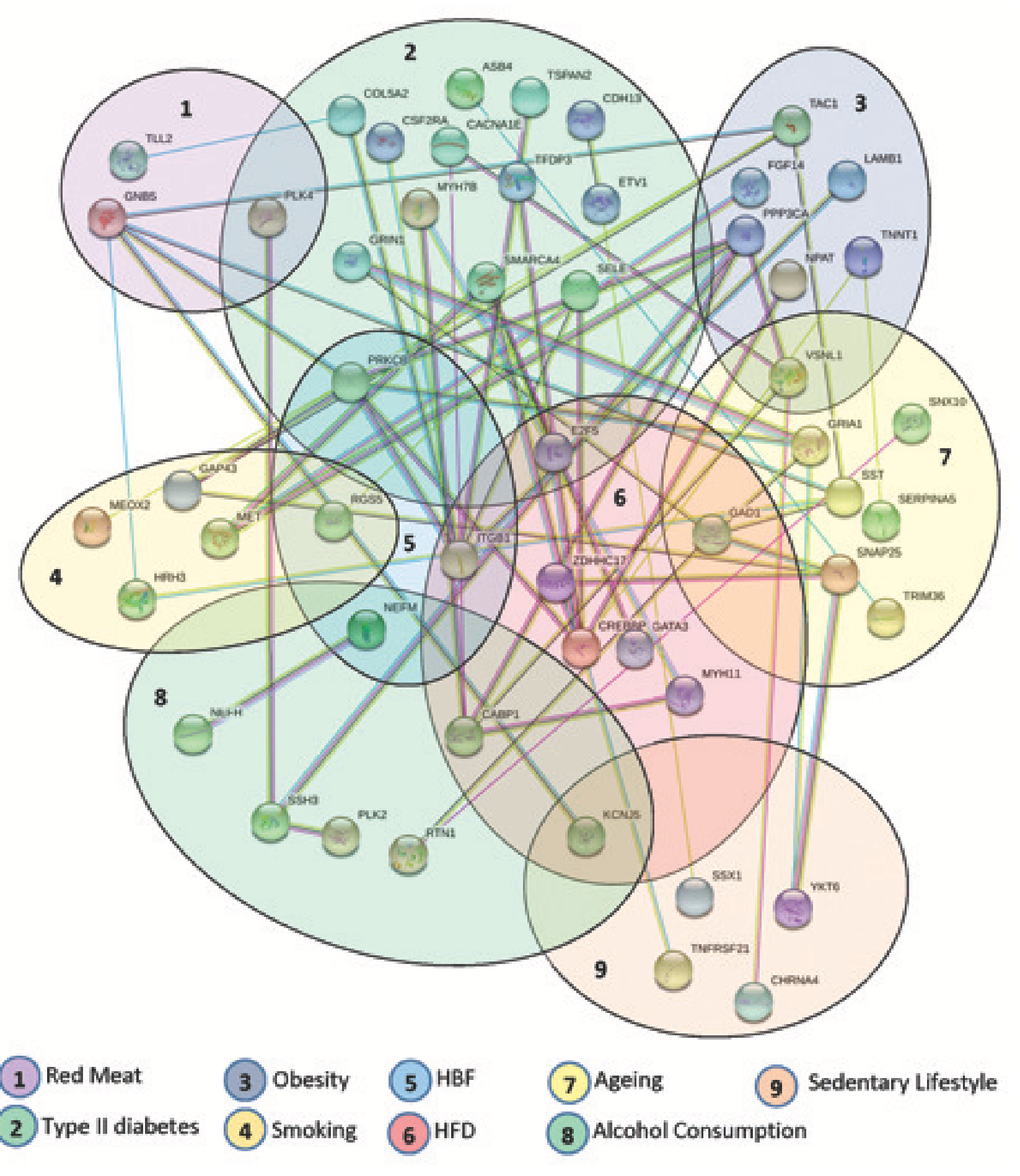

Fig. 3. Protein-Protein interaction network of commonly dysregulated genes among $\mathrm{AD}$ and other risk factors and diseases. Each cluster indicates the gene belongings.

of neurotransmitter transport (GO:0051588) and neuroepithelial cell differentiation (GO:0060563) were observed (see Table 2). A gene and gene ontology association network is constructed for the obtained gene ontology using Cytoscape (Figure-6).

\section{Discussion}

In this study, we sought novel molecular mechanisms that may affect $\mathrm{AD}$ that are made evident by genetic associations with risk factors and diseases that are known to predispose individuals to AD. For this purpose, we conducted analysis in gene expression of AD patients, molecular key pathways, gene ontologies and PPIs. These analyses that employ network-based approach can uncover novel relationships between AD and 


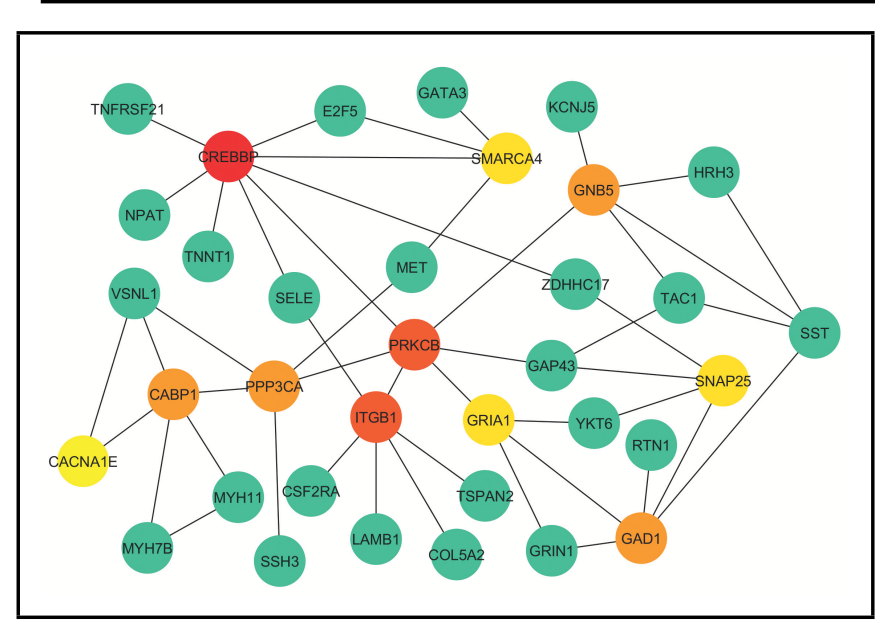

Fig. 4. The simplified PPI network of the commonly dysregulated genes among between $\mathrm{AD}$ and other risk factors and diseases. The 10 most significant hub proteins are marked as red, orange and yellow respectively.

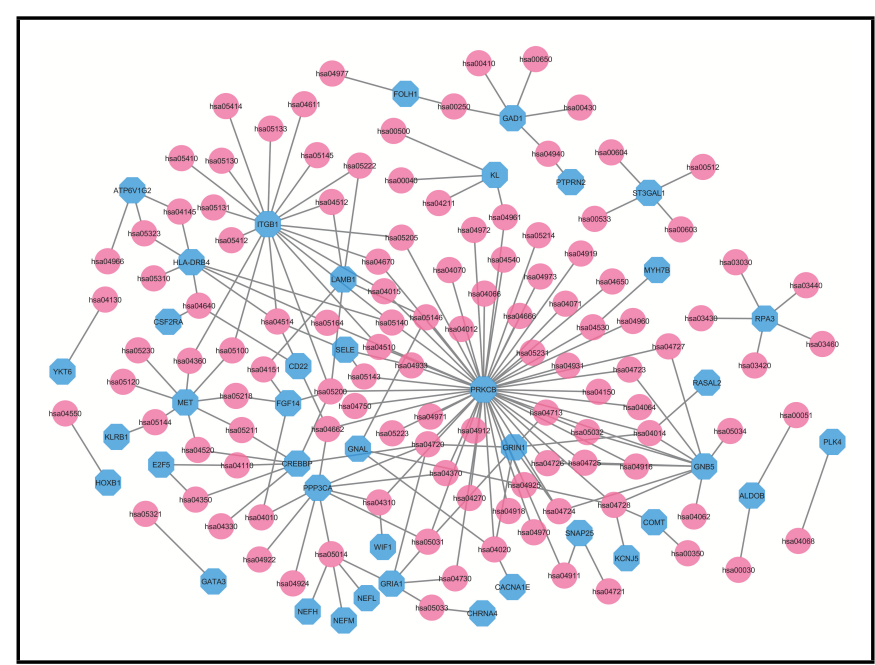

Fig. 5. The gene and pathway association network for all pathways obtained for the dysregulated genes common to the $\mathrm{AD}$ and other risk factor datasets. Sky blue-colored octagon-shaped nodes represent genes, and round-shaped pink-colored nodes represent pathway (KEGG Id). A link is placed when a gene belongs to a pathway.

other susceptibility/risk factor. The findings presented here have not been identified by any previous individual studies. Our study identified several significant genes that may be usefully investigated in other further work, and the hub genes may identify targets for therapeutic interventions in AD. Besides this, our analysis also identified and characterized a number of biological functions related to these genes that throw light on processes that lead to $\mathrm{AD}$.

Our gene expression analysis showed that the $\mathrm{AD}$ is strongly associated with type II diabetes ( 35 genes), alcohol consumption (34 genes), sedentary lifestyle (18 genes) and ageing (15 genes) as they share the maximum dysregulated genes. We constructed and analyzed the PPI network to have a better understanding of the central mechanism behind the AD. For this reason, to construct a PPI network around the differentially expressed genes for our study, we have combined the results of statistical analyses with the protein interactome network. For finding central proteins (i.e., hubs), topological analysis strategies were employed. These identified Hubs proteins might be considered as candidate biomarkers or potential
Table 2. Significant GO ontologies that are related to nervous system, and common between the $\mathrm{AD}$ and other risk factors and diseases. (Ag=Ageing, T2D=Type II Diabetes, Ob=Obesity, Sm=Smoking,RM=Red Meat, $\mathrm{AC}=$ Alcohol Consumption, $\mathrm{SL}=$ Sedentary Lifestyle.)

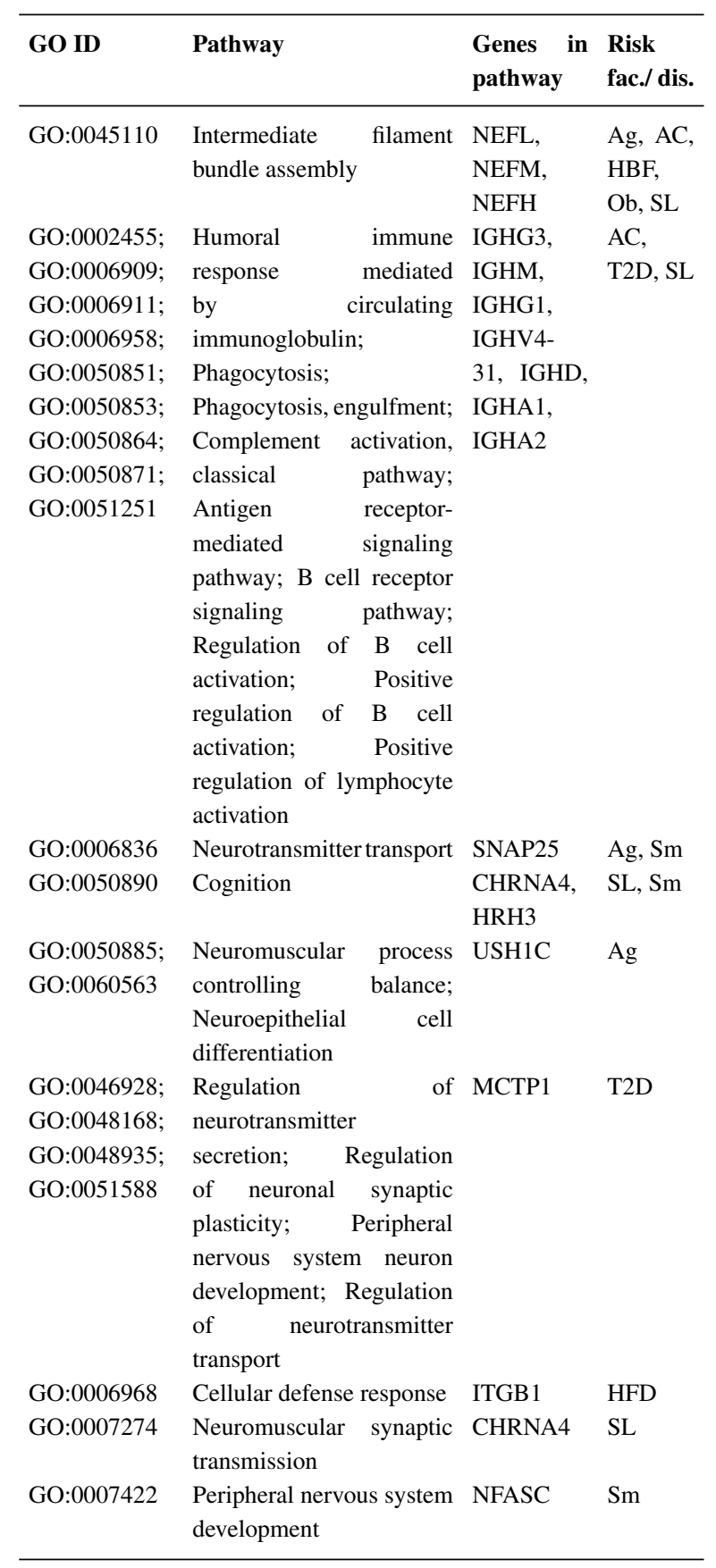

drug targets. From the PPI network analysis, it is observed that 10 hub genes (CREBBP, PRKCB, ITGB1, GAD1, GNB5, PPP3CA, CABP1, SMARCA4, SNAP25 and GRIA1) are involved in the AD.

In addition, disease-related genes play a vital role in the human interactomes via the pathways. In this study, we identified nine significant pathways that are associated with the nervous system which include Long-term potentiation, Synaptic vesicle cycle, Retrograde endocannabinoid signaling, Glutamatergic synapse, Cholinergic synapse, Serotonergic synapse, GABAergic synapse, 


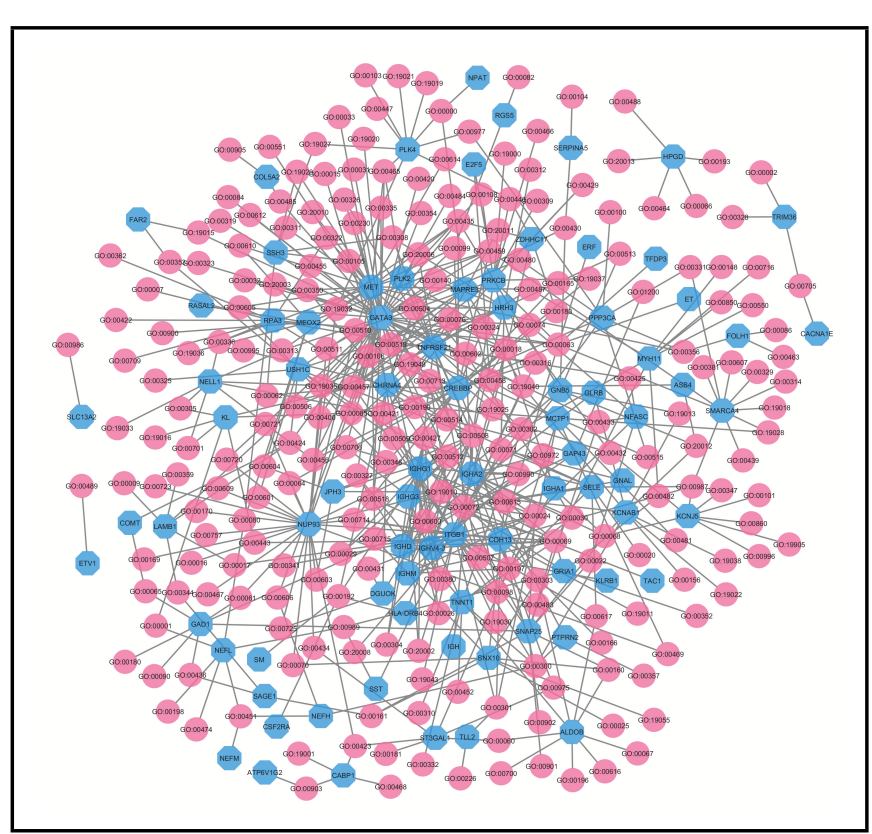

Fig. 6. The gene and gene ontology association network for all the gene ontologies obtained for commonly dysregulated genes between the $\mathrm{AD}$ and other risk factors and diseases. Sky blue-colored octagon-shaped nodes represent genes, and round-shaped pink-colored nodes represent gene ontology (GO Term). A link is placed when a gene belongs to an ontology.

Dopaminergic synapse, and Long-term depression. Our study also identified several gene ontology groups including peripheral nervous system neuron development, neurotransmitter transport, neuromuscular synaptic transmission, peripheral nervous system development, negative regulation of neurological system process, regulation of neurotransmitter secretion, regulation of neuronal synaptic plasticity, autonomic nervous system development, sympathetic nervous system development, neuromuscular process controlling balance, neuron apoptotic process, regulation of neurotransmitter transport and neuroepithelial cell differentiation. It can be noted that many of these are closely related to the nervous system.

We have also analyzed the differentially expressed genes of each risk factor and type II diabetes with OMIM and dbGaP databases using EnrichR to validate our identified results using the valid gold benchmark genedisease associations Rana et al. (2018). Table 3 shows the genes of each risk factor/disease that are resulted to be associated with the AD. These results corroborate that, the differentially expressed genes of 8 risk factors and type II diabetes are responsible for the AD. As a whole, our findings potentially fill a major gap in understanding $\mathrm{AD}$ pathophysiology. They will also open up opportunities to determine the mechanic links between the $\mathrm{AD}$ and various risk factors and diseases.

\section{Conclusions}

In this study, transcriptomic data was considered to identify the genetic association of various diseasome relationships with AD. Our findings suggest that these network methods can illustrate disease progression that yields a potential advancement towards having better insight into the origin and development of the AD. Detecting the complex relationship of various risk factors with the disease may disclose novel and useful information for having a better understanding of overall mechanism as well as planning new therapeutic strategies for $\mathrm{AD}$. Using gene expression analysis may be a basis for future accurate disease diagnosis and effective treatment which can be enhanced by the approaches employed in this
Table 3. Gene-disease association analysis of differentially expressed genes of 8 risk factors and type II diabetes with AD using OMIM and dbGaP databases.

\begin{tabular}{|c|c|c|}
\hline $\begin{array}{l}\text { Risk factor/ } \\
\text { disease }\end{array}$ & Genes & $\begin{array}{l}\text { Adjusted } \\
\text { p-value }\end{array}$ \\
\hline Ageing & $\begin{array}{l}\text { PLAG1, HMGA2, DNAH11, CR1, } \\
\text { VSNL1, DCHS2, F13A1, DISC1, } \\
\text { SLC28A1 }\end{array}$ & 4.34E-02 \\
\hline $\begin{array}{l}\text { Alcohol } \\
\text { Consumption }\end{array}$ & $\begin{array}{l}\text { GNAQ, GNAS, ARNT, APBB2, } \\
\text { ADCY2, ADCY1, IGF1R, } \\
\text { MS4A6A, RTN1, ATXN1, PIEZO2, } \\
\text { ST3GAL1 }\end{array}$ & 4.72E-01 \\
\hline $\begin{array}{l}\text { Type } \\
\text { Diabetes }\end{array}$ & $\begin{array}{l}\text { AKAP13, HNF4A, HMGA2, BUB1, } \\
\text { IGF1R, CR1, DIAPH3, TENM4, } \\
\text { CADPS, NEDD9, NPAS3 }\end{array}$ & $4.66 \mathrm{E}-01$ \\
\hline $\mathrm{HBF}$ & $\begin{array}{l}\text { PTGIR, THRA, HMGA2, ADCY2, } \\
\text { PSEN1, DRD1, BUB1, DBT, } \\
\text { PIEZO2 }\end{array}$ & $1.18 \mathrm{E}-01$ \\
\hline HFD & $\begin{array}{l}\text { AKAP13, CREBBP, HNF4A, } \\
\text { HMGA2, DNAH11, COL22A1, } \\
\text { PIEZO2, RORA, GFRA2, CD33 }\end{array}$ & $5.17 \mathrm{E}-01$ \\
\hline Obesity & $\begin{array}{l}\text { RYR2, RBFOX1, VSNL1, NR2F1, } \\
\text { HMGA2 }\end{array}$ & $1.94 \mathrm{E}-01$ \\
\hline Red Meat & HFE, HMGA2, ADCY2, F13A1 & 4.41E-01 \\
\hline Sedentary & TFAP2A, HSP90AA1, CREB1, & $1.01 \mathrm{E}-01$ \\
\hline Lifestyle & $\begin{array}{l}\text { THRA, HFE, APOE, TSHR, RYR2, } \\
\text { DIAPH3, DCHS2, DBT, CLU }\end{array}$ & \\
\hline Smoking & $\begin{array}{l}\text { A2M, OPRD1, SMAD1, ACE, } \\
\text { BUB1, CNTNAP2 }\end{array}$ & $8.45 \mathrm{E}-02$ \\
\hline
\end{tabular}

study. This enhancement may lead to new forms of personalized medicine for increasingly precise insights into disease detection, treatment and remediation for $\mathrm{AD}$.

\section{Acknowledgements \\ Funding}

This work has been supported by the... Text Text Text Text.

\section{References}

Altshuler, D. et al. (2008). Genetic mapping in human disease. science, 322(5903), 881-888.

Anstey, K. J. et al. (2009). Alcohol consumption as a risk factor for dementia and cognitive decline: meta-analysis of prospective studies. The American journal of Geriatric psychiatry, 17(7), 542-555.

Association, A. et al. (2017). 2017 alzheimer's disease facts and figures. Alzheimer's \& Dementia, 13(4), 325-373.

Bertram, L. et al. (2010). The genetics of alzheimer disease: back to the future. Neuron, 68(2), 270-281.

Blalock, E. M. et al. (2004). Incipient alzheimer's disease: microarray correlation analyses reveal major transcriptional and tumor suppressor responses. Proceedings of the National Academy of Sciences, 101(7), 2173-2178.

Büttner, P. et al. (2007). Gene expression profiles of t lymphocytes are sensitive to the influence of heavy smoking: a pilot study. Immunogenetics, 59(1), 37-43.

Calimlioglu, B. et al. (2015). Tissue-specific molecular biomarker signatures of type 2 diabetes: an integrative analysis of transcriptomics 
“output" — 2018/12/5 - 2:26 — page 8 - \#8

and protein-protein interaction data. Omics: a journal of integrative biology, 19(9), 563-573.

Chen, S.-H. et al. (2009). cyto-hubba: A cytoscape plug-in for hub object analysis in network biology. In 20th International Conference on Genome Informatics.

Duthey, B. (2013). Background paper 6.11: Alzheimer disease and other dementias. A Public Health Approach to Innovation, pages 1-74.

Feldman, I. et al. (2008). Network properties of genes harboring inherited disease mutations. Proceedings of the National Academy of Sciences, $\mathbf{1 0 5}(11), 4323-4328$

Goh, K.-I. et al. (2007). The human disease network. Proceedings of the National Academy of Sciences, 104(21), 8685-8690.

Hebels, D. G. et al. (2011). N-nitroso compound exposure-associated transcriptomic profiles are indicative of an increased risk for colorectal cancer. Cancer letters, 309(1), 1-10.

Herse, F. et al. (2007). Dysregulation of the circulating and tissue-based renin-angiotensin system in preeclampsia. Hypertension, 49(3), 604611.

Hossain, M. A. et al. (2018a). Identification of ovarian cancer gene expression patterns associated with disease progression and mortality. bioRxiv, page 473165 .

Hossain, M. A. et al. (2018b). Network-based genetic profiling, and therapeutic target identification of thyroid cancer. bioRxiv, page 480632

Janson, J. et al. (2004). Increased risk of type 2 diabetes in alzheimer disease. Diabetes, 53(2), 474-481.

Kakehi, S. et al. (2015). Increased intramyocellular lipid/impaired insulin sensitivity is associated with altered lipid metabolic genes in muscle of high responders to a high-fat diet. American Journal of PhysiologyEndocrinology and Metabolism, 310(1), E32-E40.

Kivipelto, M. et al. (2005). Obesity and vascular risk factors at midlife and the risk of dementia and alzheimer disease. Archives of neurology, 62(10), 1556-1560

Kuleshov, M. V. et al. (2016). Enrichr: a comprehensive gene set enrichment analysis web server 2016 update. Nucleic acids research, 44(W1), W90-W97.

Lage, K. et al. (2007). A human phenome-interactome network of protein complexes implicated in genetic disorders. Nature biotechnology, 25(3), 309.

Lindsay, J. et al. (2002). Risk factors for alzheimer's disease: a prospective analysis from the canadian study of health and aging. American journal of epidemiology, 156(5), 445-453.

Logue, M. W. et al. (2011). A comprehensive genetic association study of alzheimer disease in african americans. Archives of neurology, 68(12), 1569-1579.

MacLaren, R. E. et al. (2010). Association of adipocyte genes with asp expression: a microarray analysis of subcutaneous and omental adipose tissue in morbidly obese subjects. BMC medical genomics, 3(1), 3 .

Mayeux, R. and Stern, Y. (2012). Epidemiology of alzheimer disease. Cold Spring Harbor perspectives in medicine, page a006239.

McClintick, J. N. et al. (2014). Ethanol treatment of lymphoblastoid cell lines from alcoholics and non-alcoholics causes many subtle changes in gene expression. Alcohol, 48(6), 603-610.

Misu, H. et al. (2010). A liver-derived secretory protein, selenoprotein $\mathrm{p}$ causes insulin resistance. Cell metabolism, 12(5), 483-495.

Moni, M. A. and Liò, P. (2014a). comor: a software for disease comorbidity risk assessment. Journal of clinical bioinformatics, $\mathbf{4}(1), 8$.

Moni, M. A. and Liò, P. (2014b). Network-based analysis of comorbidities risk during an infection: Sars and hiv case studies. BMC bioinformatics, 15(1), 333.

Moni, M. A. and Liò, P. (2015). How to build personalized multi-omics comorbidity profiles. Frontiers in cell and developmental biology, 3, 28.
Moni, M. A. and Lio', P. (2017). Genetic profiling and comorbidities of zika infection. The Journal of infectious diseases, 216(6), 703-712.

Moni, M. A. et al. (2014). Cytocom: a cytoscape app to visualize, query and analyse disease comorbidity networks. Bioinformatics, 31(6), 969971.

Moni, M. A. et al. (2018). Early detection of neurological dysfunction using blood cell transcript profiles. bioRxiv, page 483016.

Morris, M. C. et al. (2003). Dietary fats and the risk of incident alzheimer disease. Archives of neurology, 60(2), 194-200.

Radom-Aizik, S. et al. (2005). Effects of aerobic training on gene expression in skeletal muscle of elderly men. Medicine and science in sports and exercise, 37(10), 1680-1696.

Rahman, M. H. et al. (2018a). Genetic effect of type 2 diabetes to the progression of neurological diseases.

Rahman, M. R. et al. (2018b). Blood-based molecular biomarker signatures in alzheimer's disease: Insights from systems biomedicine perspective. bioRxiv, page 481879.

Rahman, M. R. et al. (2018c). Common molecular biomarker signatures in blood and brain of alzheimers disease. bioRxiv, page 482828 .

Rana, H. K. et al. (2018). Genetic effects of welding fumes on the progression of neurodegenerative diseases. bioRxiv, page 480806

Raue, U. et al. (2012). Transcriptome signature of resistance exercise adaptations: mixed muscle and fiber type specific profiles in young and old adults. Journal of applied physiology, 112(10), 1625-1636.

Rouaux, C. et al. (2004). Targeting creb-binding protein (cbp) loss of function as a therapeutic strategy in neurological disorders. Biochemical pharmacology, 68(6), 1157-1164.

Rzhetsky, A. et al. (2007). Probing genetic overlap among complex human phenotypes. Proceedings of the National Academy of Sciences, 104(28), 11694-11699.

Sakib, N. et al. (2018). A system biology approach to identify the genetic markers to the progression of parkinson's disease for aging, lifestyle and type 2 diabetes. bioRxiv, page 482760

Satu, M. S. et al. (2018). Bioinformatics approach to identify diseasome and comorbidities effect of mitochondrial dysfunctions on the progression of neurological disorders. bioRxiv, page 483065 .

Serrano-Pozo, A. et al. (2011). Neuropathological alterations in alzheimer disease. Cold Spring Harbor perspectives in medicine, 1(1), a006189.

Seshadri, S. et al. (2010). Genome-wide analysis of genetic loci associated with alzheimer disease. Jama, 303(18), 1832-1840.

Smoot, M. E. et al. (2010). Cytoscape 2.8: new features for data integration and network visualization. Bioinformatics, 27(3), 431-432.

Suthram, S. et al. (2010). Network-based elucidation of human disease similarities reveals common functional modules enriched for pluripotent drug targets. PLoS computational biology, 6(2), e1000662.

Szklarczyk, D. et al. (2016). The string database in 2017: quality-controlled protein-protein association networks, made broadly accessible. Nucleic acids research, page gkw937.

Tilley, L. et al. (1998). Genetic risk factors in alzheimer's disease. Molecular Pathology, 51(6), 293.

Torkamani, A. et al. (2008). Pathway analysis of seven common diseases assessed by genome-wide association. Genomics, 92(5), 265-272.

Waring, S. C. and Rosenberg, R. N. (2008). Genome-wide association studies in alzheimer disease. Archives of neurology, 65(3), 329-334.

$\mathrm{Xu}$, H. et al. (2015). Network regularised cox regression and multiplex network models to predict disease comorbidities and survival of cancer. Computational biology and chemistry, 59, 15-31.

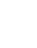

